1 Post Graduate Program in Oral Science, Prosthodontic Unit, Federal University of Santa Maria, Santa Maria, Rio Grande do Sul, Brazil

${ }^{2}$ Department of Chemical Engineering, Federal University of Santa Maria, Santa Maria, Rio Grande do Sul, Brazil.

a Physics Department, Federal University of Santa Maria, Santa Maria, Rio Grande do Sul Brazil.(Isdorneles@gmail.com; +55-55-98131-5938)
Corresponding author:

Liliana Gressler May

Federal University of Santa Maria Address: Rua Marechal Floriano Peixoto, 1184, CEP 97015-372, Centro, Santa Maria, RS, Brazil. Phone: +55-55-3220-9276, Fax: +55-55-3220-9272

E-mail: liligmay@gmail.com

Received: May 02, 2018

Accepted: December 18, 2018

\section{Y-TZP surface treatments and their effects on the bond strength to resin cement}

\author{
Michele Mirian May ${ }^{1}$, Ana Maria Estivalete \\ Marchionatti ${ }^{1}$, Luiz Felipe Valandro ${ }^{1}$, Edson Luiz \\ Foletto ${ }^{2}$, Lucio Strazzabosco Dorneles ${ }^{3}$, Liliana \\ Gressler May ${ }^{1, *}$
}

Aim: This study evaluated the effect of surface treatments of yttria-stabilized tetragonal zirconia polycrystal (Y-TZP) ceramics on their bond strength to a resin cement. Methods: Seventy zirconia blocks ( $6 \times 6 \times 2 \mathrm{~mm}^{3}$, IPS e.max ZirCAD) were assigned into 7 groups ( $n=10)$ - as-sintered(AS), no treatment; tribochemical silica coating + silanization (TBS; Cojet-sand; ProSil); air abrasion with $45 \mu$ malumina particles + universal primer (AAP; Monobond ${ }^{\circledR}$ Plus); fusion sputtering (FS); $\mathrm{SiO}_{2}$ nanofilm + silanization (SN; ProSil); FS+SN+silanization (FSSN; ProSil); FS+SN+Universal Primer (FSSNP; Monobond ${ }^{\top}$ Plus). Afterwards, a resin cement (RelyX ${ }^{\text {TM }}$ ARC) was applied inside cylinders ( $\varnothing=0.96 \mathrm{~mm} \times 1 \mathrm{~mm}$ height) placed on the zirconia surfaces. Microshear bond strength tests ( $\mu$ SBS) were carried out $(1 \mathrm{~mm} / \mathrm{min})$. Failure and phase transformation analysis were performed. Bond strength data (MPa) were subjected to Kruskal-Wallis/Mann Whitney tests. Results: TBS $(27 \pm 1.2)$ and AAP $(24.7 \pm 0.8)$ showed higher bond strengths than the other groups, followed by FSSNP (15.5 \pm 4.2$)$ and FSSN (13.3 \pm 3.6$)$. FS $(3.4 \pm 0.44)$ and SN $(9.5 \pm 2.7)$ showed the lowest values $(p<0.001)$. Most of the specimens exhibited an adhesive failure. Conclusion: Air-abrasion by silica-coated alumina particles followed by silanization or by alumina particles followed by universal primer resulted in the highest resin bond strength to zirconia. Fusion sputtering and silica nanofilm deposition induced low strengths. However, when these methods are applied in combination and with a primer (FSSN and FSSNP), higher bond strengths may be achieved. Low bond strengths are obtained when no zirconia treatment is performed.

Keywords: Adhesives. Ceramics. Yttrium. Resin Cements. 


\section{Introduction}

Interest in zirconia-based ceramics (especially yttria-stabilized tetragonal zirconia polycrystal; Y-TZP) has been increasing over the past few decades due to their excellent mechanical, aesthetic, and biocompatibility properties ${ }^{1}$. However, due to the absence of silica in there structures, these materials can neither be etched by HF nor form siloxane bonds with the silane groups in the bifunctional chemical chain ends ${ }^{2}$. Consequently, adhesion to resin cements is difficult; therefore, pretreatment of the surface to promote topographical alterations to increase micromechanical retention and provide chemical bonding to the resin cement is recommended ${ }^{3}$.

Abrasion with alumina particles is often advocated to increase the surface roughness and area of adhesion ${ }^{1}$. Alumina particle abrasion followed by cementation with resin cements containing 10-methacryloyloxydecyl dihydrogenphosphate (MDP) is one of the recommended approaches for cementing zirconia restorations $s^{1,4}$. Another widespread technique is the use of tribochemical silica coating; it involves air abrasion with aluminum oxide particles coated with silicon oxide, to promote topographical changes (micromechanical retention) and coating silicon oxide on the zirconia surface, to allow bonding with silane coupling agents ${ }^{4,5}$. This method leads to a considerable increase in the bond strength between zirconia ceramics and resin cement ${ }^{5}$.

Some researchers consider that zirconia adhesion is well understood, even though there is no consistent data on the effect of bond strength on crown retention ${ }^{6}$. Clinical studies reported a retention loss of up to $6.6 \%$ after 5 years ${ }^{7}$. Retention loss was found in 7 out of 16 studies involving zirconia cementation, considering periods of 12 and 38 months. A $20 \%$ retention loss was observed in inlay retainers cemented with composites, even with the previously mentioned tribochemical treatment of the surfaces with a Rocatec system (3M-ESPE, Seefeld, Germany) ${ }^{8}$.

Additionally, owing to the metastability of zirconia, the stresses generated during particle air abrasion may induce tetragonal to monoclinic phase transformation, which might interfere with its susceptibility to aging ${ }^{9}$. Particle abrasion on the the surface of zirconia also leads to microcracks and structural defects, which might leave the material susceptible to fracture during function ${ }^{10}$. However, this assumption is controversial because fatigue behavior improvement was observed by some authors ${ }^{11,14}$. Pereira et al. ${ }^{15}$ (2015) described that phase transformation induces a residual compressive stress and the defects take place at compressive zone solely, there is an increase in toughness. However when the defects extend beyond this zone, the transformation leads to pull out of surface's grains and increase roughness; as consequence a decrease in resistance might be expected.

From that debatable standpoint, several studies tested alternative surface pretreatments of Y-TZP ceramics to replace particle air abrasion, such as surface glazing ${ }^{16}$, primers ${ }^{3}$ and conditioning with $\mathrm{HCl}$ and $\mathrm{Fe}_{2} \mathrm{Cl}_{3}$ at $100^{\circ} \mathrm{C}^{1}$. Aboushelib ${ }^{17}$ (2012) proposed a surface treatment method involving sputtering micrometric particles of unsintered zirconia onto partially sintered machined zirconia (computer aided design - computer aided machined; CAD-CAM) and then fusing them to the surface by a sintering process. This method, known as fusion sputtering, has promised to increase the 
adhesive surface area and micromechanical retention between zirconia and the luting cements without damaging zirconia surface integrity. In addition, Druck et al. ${ }^{18}$ (2015) studied the effect of silica nanofilm plasma deposition on the bond strength between resin and $\mathrm{Y}$-TZP in terms of the chemical bonding between silica, the silane coupling agent, and resin cement, concluding that the deposition of a $5 \mathrm{~nm}$-thick film followed by silane application induced a high bond strength, similar to tribochemical treatment, without causing tetragonal-to-monoclinic transformation.

In the present in vitro study, we intend to compare different methods of surface treatment of $\mathrm{Y}$-TZP ceramics and their effects on the bond strength to a resin cement. The first hypothesis tested was that zirconia surface treatment would result in a higher bond strength when compared to untreated zirconia. The second hypothesis was that the treatments tested would yield results similar to those of the tribochemical treatment.

\section{MATERIALS AND METHODS}

The main compositions of the materials used in this study are shown in Table 1.

\section{Sample calculations}

Sample calculations were performed using data from a pilot study; the fusion sputtering method was compared with the no-treatment condition (control group). An opensource calculator, available at www.openepi.com, was used to determine the difference of averages. For a $95 \%$ confidence interval and power of $80 \%$, the estimated sample size was 5 specimens per group. A sample size of 10 specimens per group was established, so that the differences between them could be detected.

\section{Zirconia specimen preparation}

Y-TZP (IPS e.max ZirCAD for inLab®, Ivoclar-Vivadent, Schaan, Liechtenstein) was sectioned with a diamond disk using a cutting machine (Labcut 1010, Extec, Enfield,

Table 1. Compositions of the used materials

\begin{tabular}{|c|c|c|}
\hline Material & Composition & $\begin{array}{l}\text { Batch } \\
\text { Number }\end{array}$ \\
\hline $\begin{array}{l}\text { IPS e.max ZirCAD for inLabß, } \\
\text { Ivoclar-Vivadent, Schaan, } \\
\text { Liechtenstein }\end{array}$ & $\begin{array}{c}\text { Zirconium dioxide }\left(\mathrm{ZrO}_{2}\right) 87-95 \mathrm{wt} . \% \text {, yttrium oxide }\left(\mathrm{Y}_{2} \mathrm{O}_{3}\right) \\
3-4 \mathrm{w} \% \text {, hafnium oxide }\left(\mathrm{HfO}_{2}\right) 1-5 \mathrm{wt} \% \text {, and aluminum } \\
\text { oxide }\left(\mathrm{Al}_{2} \mathrm{O}_{3}\right)<1 \%\end{array}$ & S21721 \\
\hline $\begin{array}{l}3 \mathrm{M}^{\text {Tu }} \text { RelyX } \mathrm{X}^{\mathrm{tu}} \text { ARC, } 3 \mathrm{M} / \mathrm{ESPE}, \\
\text { Seefeld, Germany }\end{array}$ & $\begin{array}{l}\text { Bisphenol-A-diglycidylether dimethacrylate (BisGMA) } \\
\text { and triethylene glycol dimethacrylate (TEGDMA) polymer, } \\
\text { zirconia/silica filler> } 67.5 w t . \% \text {, dimethacrylate polymer, } \\
\text { benzoyl peroxide, amine, photoinitiator, and pigment }\end{array}$ & 1416400377 \\
\hline $\begin{array}{l}\text { Aluminium Trioxide, } \\
\text { POLIDENTAL, Cotia, Brazil }\end{array}$ & $45 \mu \mathrm{m}$ aluminum oxide particles & 44493 \\
\hline Cojet-Sand, 3M/ESPE & $30 \mu \mathrm{m}$ aluminum oxide particles coated with silicon oxide & 524851 \\
\hline $\begin{array}{l}\text { Monobond }{ }^{\circledR} \text { Plus, Ivoclar-Vivadent, } \\
\text { Schaan, Liechtenstein }\end{array}$ & $\begin{array}{c}\text { 3-trimethoxysilylpropyl methacrylate } \leq 2.5 \% \text {, ethanol } \\
50 \%-100 \% \text {, methacrylated phosphoric acid ester } \\
\leq 2.5 \% \text {,and sulfide methacrylate }\end{array}$ & S02028 \\
\hline ProSil, FGM, Joinville, Brazil & $\begin{array}{c}<5 w t . \% \text { of 3-methacryloxypropyltrimethoxy-silane, } \\
>97 \text { wt. } \% \text { ethanol, and }<10 \text { wt.\% water }\end{array}$ & 130314 \\
\hline
\end{tabular}


USA) to yield 70 blocks $\left(6 \times 6 \times 2 \mathrm{~mm}^{3}\right)$. After cutting, the surfaces of the blocks were standardized manually with a 1200 grit sandpaper ${ }^{18}$. The specimens were randomly (random.org) allocated into 7 groups $(n=10)$ according to the type of surface treatment, as listed below.

\section{Zirconia surface treatments}

- As-sintered (AS) (control group): Zirconia blocks were sintered as recommended by the manufacturer and received no surface treatment prior to cementation.

- Tribochemical silica coating (TBS): After sintering, the specimens were subjected to tribochemical treatment - air abrasion with alumina particles coated with silica $(30 \mu \mathrm{m})($ CoJet-Sand $\AA$, 3M ESPE, Seefeld, Germany) at a distance of $10 \mathrm{~mm}$ perpendicular to the specimen and pressure of 2.8 bar for $10 \mathrm{~s}$. A device was used to standardize the application ${ }^{6}$. The silane coupling agent (ProSil, FGM, Joinville, Brazil) was applied with a disposable micro applicator and the specimens were left undisturbed for $5 \mathrm{~min}$ for solvent evaporation before cementation. The TBS group served as the positive control.

- Abrasion with alumina particles (AAP) followed by primer application: After sintering, the zirconia surfaces were abraded with aluminum oxide particles $(45 \mu \mathrm{m})$ at a pressure of 2.8 bar; the rest of the parameters were similar to those of the TBS group. Afterwards, the treated surfaces were coated with the Monobond ${ }^{\oplus}$ Plus primer (Ivoclar-Vivadent) using a disposable micro applicator over $60 \mathrm{~s}$. This primer is an alcoholic solution of 3-methacryloxypropyl-trimethoxysilane, phosphoric acid methacrylate, and sulfide methacrylate (containing multiple bond promoters). The AAP group followed the surface treatment protocols recommended by the ceramic manufacturer.

- Fusion sputtering (FS): Prior to sintering, a 50\% ethyl alcohol suspension containing microparticles of zirconia powder was sprayed onto zirconia surfaces. This method was adapted from the protocol proposed by Aboushelib ${ }^{17}$, as described below. Y-TZP powder was obtained by milling pre-sintered blocks of the ceramic used for CAD/CAM machining of prosthetic restorations. Firstly, the pre-sintered blocks were crushed and transferred into a sealed capsule containing alumina spheres, which was then placed in a ball mill (MA500, Marconi Laboratory Equipment, Piracicaba, Brazil). Using rapid circular movements in the horizontal direction of the capsule on the motorized rollers, the sintered alumina spheres were directed away from their inner walls for $2 \mathrm{~h}$ to pulverize the samples. The pulverized materials were sieved through 325 stainless steel meshes, allowing the passage of particles smaller than $45 \mu \mathrm{m}$ (sieve for granulometric analysis, BERTEL Metal industry, Caieiras, Brazil).

The obtained powders $(10 \mathrm{~g})$ were mixed with $10 \mathrm{~mL}$ of ethyl alcohol $(50 \%)$ and treated in an ultrasonic vibrator to homogenize the particle distribution. Immediately after mixing, the solution was transferred to a glass vessel attached to an airbrush (used for spray painting). To standardize the distance and area of application, the same device as that used to standardize the blasting of particles in the TBS and AAP groups was used. At first, small jets were dispensed on a black paper until a homogeneous 
mixture was observed. Then, the zirconia specimens were sprayed for $5 \mathrm{~s}$ at 3 bar and stored at $60^{\circ} \mathrm{C}$ for $2 \mathrm{~h}$ prior to sintering, as prescribed by the manufacturer.

- $5 \mathrm{~nm} \mathrm{SiO} 2$ nanofilm (SN) deposition: After sintering, 5 nm-thick silica nanofilms were deposited on zirconia surfaces as proposed by Druck et al. ${ }^{18}$ (2015), using a plasma deposition process via magnetron sputtering. The process was as follows: The specimens were initially fixed in a sample holder located inside a chamber containing a silica target and a plasma cannon. The chamber was closed and the atmosphere inside it was pumped down to $10^{-5} \mathrm{~Pa}$ in order to achieve ideal vacuum for deposition. Then, argon was admitted into the chamber at a flow rate of $20 \mathrm{sccm}$ (standard cubic centimeters per minute) and $0.69 \mathrm{~Pa}$. Argon atoms act as cathodes being attracted by the anode, i.e., the plasma cannon. Thus, the silica target was bombarded by the argon atoms and released silica inside the chamber, which was then deposited on all the surfaces. For the deposition of a $5 \mathrm{~nm}$-thick film, a deposition time of $120 \mathrm{~s}$ is required. Prior to cementation, the specimens were coated with a silane coupling agent (ProSil FGM, Dentscare LTDA, Joinville, SC, Brazil) using a disposable micro applicator and were left undisturbed for 5 min for solvent evaporation.

- Fusion sputtering $+\mathrm{SiO}_{2}$ nanofilm (FSSN) deposition: The FSSN group is a combination of the FS and SN treatments. In the first step, the FS treatment was performed followed by sintering of the zirconia blocks and the $\mathrm{SN}$ treatment $\left(5 \mathrm{~nm} \mathrm{SiO}{ }_{2}\right.$ nanofilm deposition and silanization) was carried out.

- Fusion sputtering + $5 \mathrm{~nm} \mathrm{SiO}{ }_{2}$ nanofilm + primer (FSSNP): The FSSN treatment was firstly performed followed by the application of the Monobond ${ }^{\oplus}$ Plus (Ivoclar-Vivadent) primer, with a disposable micro applicator over $60 \mathrm{~s}$.

\section{Adhesive procedure}

Each Y-TZP block was embedded in a plastic cylinder ( $h=14 \mathrm{~mm}, \varnothing=25 \mathrm{~mm}$ ) with a chemically activated acrylic resin, which protected the bonding surface from contamination. Afterwards, a technique adapted from Tedesco et al. ${ }^{19}(2013)$ was carried out as follows. Four starch tubes of $1 \mathrm{~mm}$ height and $0.96 \mathrm{~mm}$ internal diameter were placed on the previously treated zirconia surfaces of each group, after which a resin cement (RelyX ARC, 3M ESPE, Seefeld, Germany) was poured into these tubes using an endodontic instrument (digital spacer size A, $25 \mathrm{~mm}$, Dentsply Maillefer). A glass slide was pressed to the tops of the tubes and photo-activation was performed for 40 s (Radii-cal, SDI, Bayswater, Australia).

The specimens were stored in distilled water at $37^{\circ} \mathrm{C}$ for $24 \mathrm{~h}$. Later, the tubes were dissolved by immersion in water for $24 \mathrm{~h}$ and removed using an exploratory probe. Stereomicroscope analysis with 35x magnification was performed (Stereomicroscope Discovery V20, Carl Zeiss, Göttingen, Germany) to observe defects in the adhesive interface. If gaps at the margins in the adhesive zone, air bubbles, or other defects were detected, the microcylinders were excluded from statistical analysis.

The area of the adhesive interface $A\left(\mathrm{~mm}^{2}\right)$ was calculated from the cross-sectional area of the cylinder, $A=\pi r^{2}$, where $r$ is the radius of the obtained circle, and was found to be $0.72 \mathrm{~mm}^{2}$. 


\section{Microshear bond test}

Microshear tests were executed shortly after removing the starch tubes. The specimens were mounted on a specific device and fixed in a universal testing machine (EMIC DL1000, Emic, São José dos Pinhais, Brazil). A stainless steel wire $(0.3 \mathrm{~mm}$ in diameter) was placed close to the free surface of zirconia in contact with the lower semicircle of the resin cement microcylinder; subsequently, a shear load was applied at a rate of $1 \mathrm{~mm} / \mathrm{min}$ until failure occurred.

The bond strength $R(\mathrm{MPa})$ was calculated using the equation $R=F / A$, where $F$ is the load for failure of the specimen $(N)$ and $A$ is the area of the adhesive interface $\left(0.72 \mathrm{~mm}^{2}\right)$.

\section{Failure mode analysis}

All the specimens were analyzed stereomicroscopically at 10x-60x magnification to identify the type of failure (Stereomicroscope Discovery V20, Carl Zeiss).

The failure modes were classified as follows. 1) Adhesive: failure at the cement/ceramic interface, no cement residues present on the ceramic surface; 2) Predominantly adhesive: The adhesive failure took place in more than $50 \%$ of the area, but there were remnants of cement on the cementation surface.

In addition, representative fractures of each group were selected and analyzed by SEM (scanning electron microscopy; Jeol-JSM-T330A, Jeol Ltd., Tokyo, Japan).

\section{X-ray diffraction}

X-ray diffraction (XRD) analysis was performed on the surface of treatment-free Y-TZP as well as samples from each of the 6 treatment groups (D8 Advanced XDR, Bruker AXS GmbH, Germany). The patterns were obtained in the $2 \theta$ range of $20^{\circ}$ to $34.988^{\circ}$ at a step size $0.02^{\circ}$ with 175 s per step. The source of radiation was CuKa $(\lambda=1.5416 \AA)$.

The amount of monoclinic phase $(\mathrm{Xm})$ was calculated according to the Garvie and Nicholson ${ }^{20}(1972)$ formula:

$$
X m=\frac{\operatorname{Im}(-111)+\operatorname{Im}(111)}{\operatorname{Im}(111)+\operatorname{Im}(-111)+\operatorname{It}(101)}
$$

where $I_{m}(-111)$ and $I_{m}(111)$ correspond to the low intensities of monoclinic phase peaks $\left(2 \theta \sim 28^{\circ}\right.$ and $31.2^{\circ}$, respectively) and $\mathrm{I}_{\mathrm{t}}(101)$ corresponds to the intensity of the tetragonal phase peak $\left(2 \theta \sim 30^{\circ}\right)$.

The volumetric fraction $F_{m}$ of the monoclinic phase was calculated as described by Toraya et al. ${ }^{21}$ :

$$
F m=\frac{1.311 X m}{1+0.311 \times m}
$$

\section{Micromorphological analysis}

Micromorphological analysis of one sample per treatment group was performed using a SEM (Jeol-JSM-T330A, Jeol Ltd, Tokyo, Japan) at 200x to 2000x magnification. 


\section{Data analysis}

For statistical analysis, those microcylinders that exhibited pre-test failure were excluded and the mean of the remainders was calculated per zirconia block (considered as sample unit). Subsequently, the mean and standard deviation of each study group were calculated. The final sample size was not changed $(n=10)$, except in the AS group where analysis were carried out using results from two specimens $(n=2)$, since high pre-test failure occurred.

As the assumptions of normality of distribution and homogeneity of variances were not found even after data transformation, the non-parametric Kruskal-Wallis test was performed for all groups $(p<0.001)$. Later, multiple Mann-Whitney tests were performed in pairs to verify the statistical differences between different groups.

\section{RESULTS}

The Kruskal-Wallis test indicated statistically significant differences between different types of treatments $(p<0.001)$.

The TBS and AAP groups showed the highest bond strengths, followed by the FSSN, FSSNP, FS, and SN groups (Table 2). All the treatments resulted in higher bond strengths when compared to the as-sintered conditions. Therefore, the first hypothesis (different surface treatments would lead to a higher bond strength than when compared to untreated zirconia) was accepted.

The second hypothesis (treatments would promote bond strengths in a manner similar to the tribochemical treatment) was partially rejected. The FS, SN, FSSN, and FSSNP treatments resulted in lower bond strengths than the positive control (TBS), while the AAP treatment was similar to TBS (Table 2).

Adhesive failures were predominant in the AS and FS groups (Table 2). The TBS, AAP, SN, FN, and FSSNP groups exhibited predominantly adhesive failures, i.e., with visible cement remnants on the ceramic surface, usually at the margins of the microcylinders, over less than $50 \%$ of the area (Figure 1).

Table 2. Bond strength (MPa) of Y-TZP with the resin cement depending on the surface treatment. XRD analysis results (\% of monoclinic phase). Classification of the failure modes after microshear bond strength tests

\begin{tabular}{lcccc}
\hline \multirow{2}{*}{ Group } & Bond Strength* $(\mathrm{MPa})$ & Monoclinic Phase $(\%)$ & \multicolumn{2}{c}{ Failure Mode (\%) } \\
\cline { 3 - 5 } & $0.19(0.16)^{\mathrm{E}}$ & 7.53 & Adhesive & Mainly Adhesive \\
\hline AS & $26.97(1.21)^{\mathrm{A}}$ & 17.17 & 100 & 0 \\
\hline TBS & $24.67(0.8)^{\mathrm{A}}$ & 18.00 & 10 & 90 \\
\hline AAP & $3.37(0.44)^{\mathrm{D}}$ & 11.05 & 100 & 0 \\
\hline FS & $9.53(2.67)^{\mathrm{C}}$ & 5.05 & 40 & 60 \\
\hline SN & $13.26(3.58)^{\mathrm{B}}$ & 5.16 & 8 & 92 \\
\hline FSSN & $15.50(4.21)^{\mathrm{B}}$ & - & 18 & 82 \\
\hline FSSNP & &
\end{tabular}

* The letters in the superscripts indicate statistical differences between different groups 
XRD analysis (Table 2; Figure 2) indicated the presence of monoclinic contents on the zirconia surfaces of all the groups, including AS.

The micrographs (Figure 3 ) revealed differences in the topographie of the samples treated by different methods. The blasting protocols resulted in similar grooves at the micrometer scale. Fusion sputtering resulted in retentive grains, which can be observed in Figure 3 (images E-F). At higher magnifications, we observed contours of zirconia grains on the surfaces as well as fused agglomerates (Figure 3, image F).

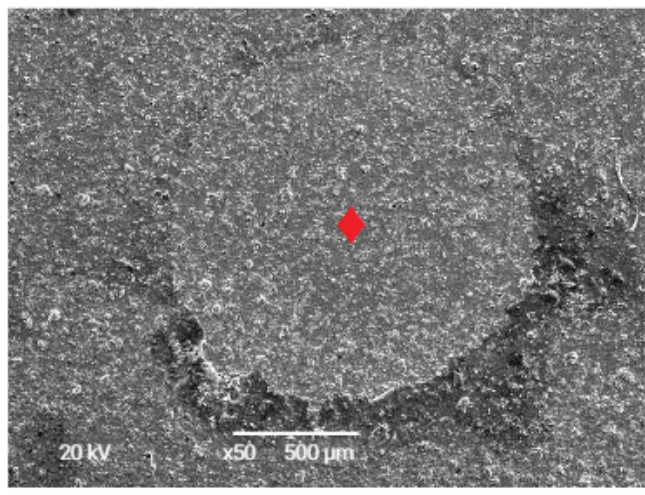

A

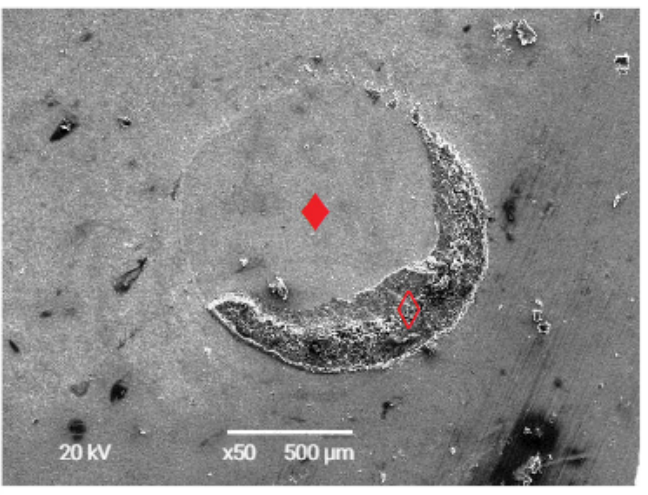

B

Figure 1. Representative micrographs (SEM) of the failure modes after the microshear test. A presents the adhesive failure mode at the ceramic/cement interface in a FS group specimen while B demonstrates the predominantly adhesive failure mode in a specimen of the TBS group. The symbol ( $\bullet$ represents the ceramic surface free of cement, while the symbol $(\diamond)$ represents the surface with the presence of resin cement.

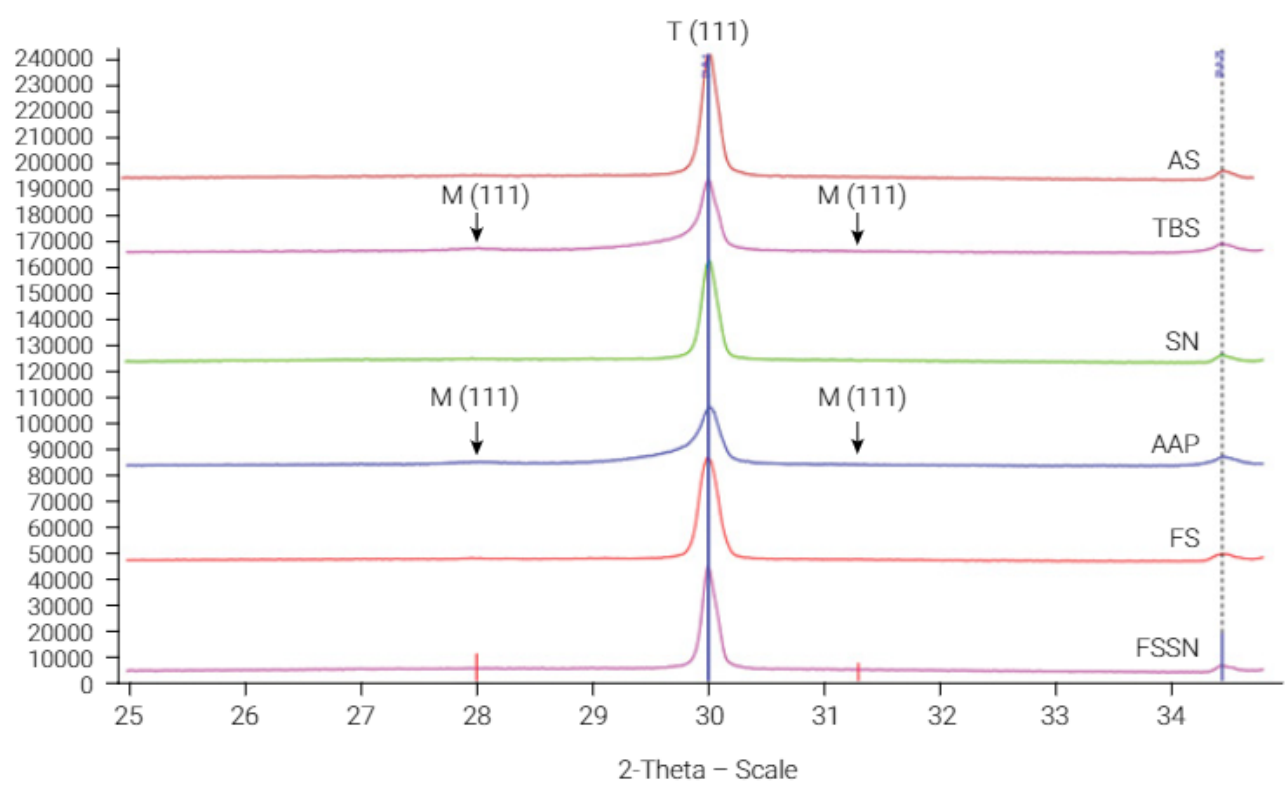

Figure 2. Diffraction analyses - The location of the peaks was compared to the values available for tetragonal zirconium and monoclinic zirconium in the spectra of the diffraction pattern maintained by the International Center Diffraction Data/Joint Committee for Powder Diffraction Studies, represented by the symbols M $(-111)$ and (111) (monoclinic peaks), $2 \theta \approx 28^{\circ}$ and $2 \theta \approx 31.2^{\circ}$, respectively, and T (111) (tetragonal), $2 \theta \approx 30^{\circ}$. 


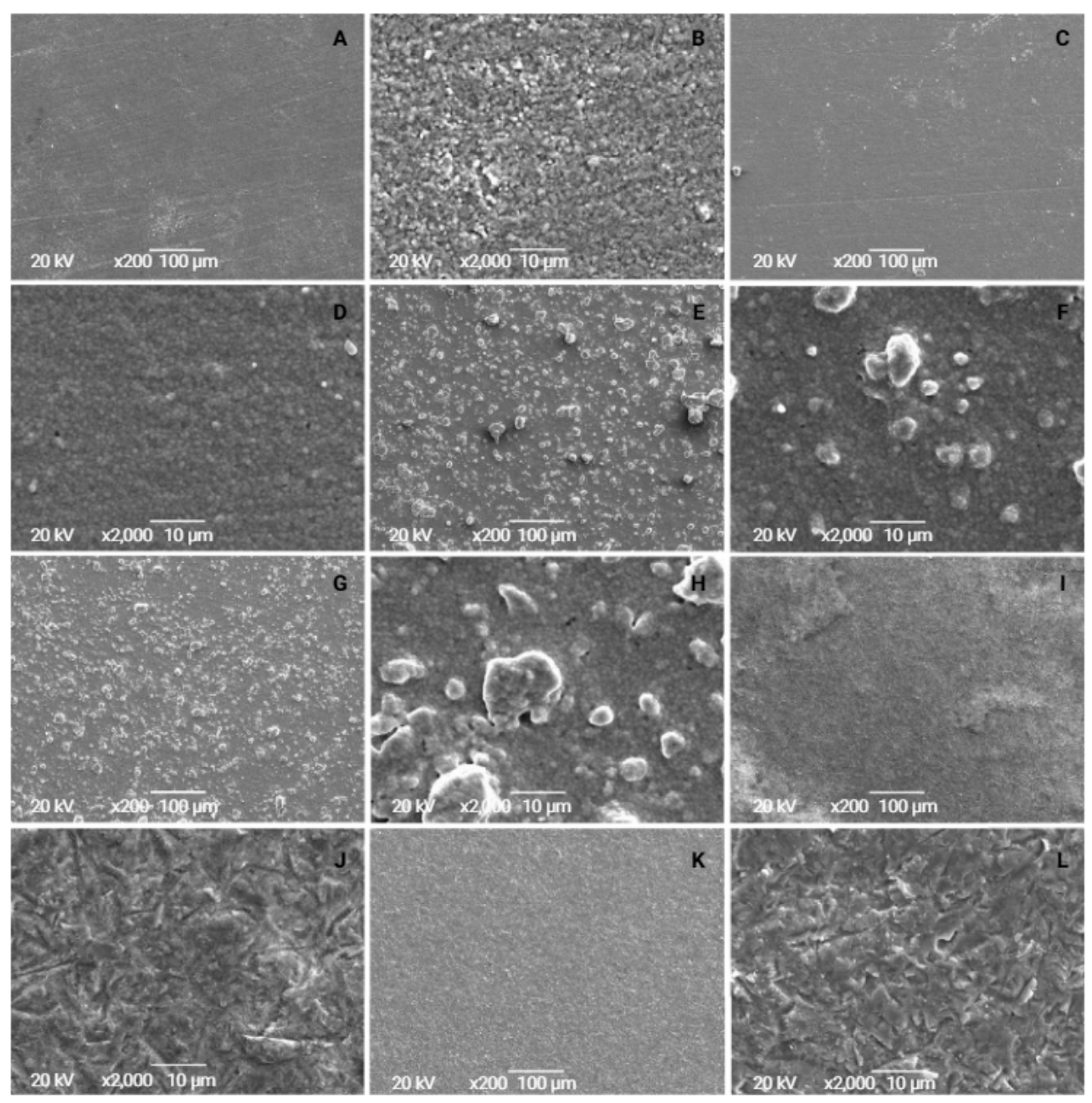

Figure 3. Representative micrographs of the surface of Y-TZP. A-B) no treatment; C-D) nanofilm deposition; E-F) fusion sputtering; G-H) fusion sputtering followed by silica deposition; I-J) tribochemical treatment; $\mathrm{K}-\mathrm{L})$ abrasion with alumina particles.

The deposition of nanofilms does not seem to have modified the topography when compared to the specimens on which nanofilms were not deposited (AS and FS).

\section{DISCUSSION}

The findings of this study showed that the AAP zirconia surface treatment resulted in a bond strength similar to that of the TBS treatment. The AAP group adopted the manufacturer's recommendations for zirconia treatment (IPS e.max ZirCAD), which dictates air abrasion with alumina particles (maximum of $50 \mu \mathrm{m}$ ) followed by the application of a universal primer containing phosphoric acid methacrylate ester as the functional monomer (Monobond(B)Plus, Ivoclar Vivadent).

These results are in agreement with those of Perdigão et al. ${ }^{9}$ (2013) and Inokoshi et al. ${ }^{22}$ (2014) In a meta-analysis on the effectiveness of zirconia adhesion, Inokoshi et al. ${ }^{22}$ 
(2014) reported an increase in the bond strength between a ceramic and resin cement when the ceramic was subjected to $\mathrm{Al}_{2} \mathrm{O}_{3}$ abrasion or tribochemical silica coating. A positive effect was observed on the adhesion to Y-TZP and bond stability when abrasion with $\mathrm{Al}_{2} \mathrm{O}_{3}$ was followed by the application of a primer containing MDP.

A controversial aspect regarding particle air abrasion of zirconia surfaces is the effect of this treatment on their mechanical properties. Some authors report that the tetragonal to monoclinic phase transformation induced by abrasion increases the flexural strength of zirconia by creating a surface area containing a residual compressive stress $^{14}$. Other studies report that the presence of monoclinic contents can result in superficial changes with the creation of structural defects that make zirconia susceptible to hydrothermal degradation and radial cracks during operation ${ }^{1,10}$. Also, the particles air-abrasion might create grooves and micro defects as a result of the high impact energies of the particles. Sharp grooves and a superficial chipping pattern (Figure 3, images I-J and K-L) were observed. Several studies report that the extent of damage is not limited to the surface only, but also extends from 30 to $50 \mu \mathrm{m}$ of the subsurface depth of the material, resulting in grain loss and microcracks, which might significantly damage the mechanical behavior of the material ${ }^{10}$.

On the other hand, some studies reported that particle air abrasion does not impair the fatigue behavior of zirconia materials ${ }^{11,14}$. Other studies have also found that the mechanical fatigue behavior of zirconia crowns is not affected, even when the inner surfaces of zirconia crowns were abraded with alumina particles before cementation ${ }^{12,13}$.

In the current study, even in the AS group, some monoclinic content was found (Table 2). According to Subaşi et al. ${ }^{23}$ (2014), monoclinic contents may be dependent on the type of material. Different amounts of monoclinic content were found in the control groups in other studies as well ${ }^{23,24}$. In the AAP, TBS, and FS groups, there was an increase in the monoclinic content $(10.47 \%, 9.64 \%$, and $3.52 \%$, respectively) as compared to the AS group (Figure 2). Pereira et al. ${ }^{15}$ (2015) found that a monoclinic phase content greater than $50 \%$ is necessary to induce a decrease in the flexural strength. Therefore, it is to be inferred that the treatments tested in the present study do not adversely affect the structural properties of Y-TZP.

Alternatively, the fusion sputtering method (FS) has been shown to be facile and inexpensive to conduct ${ }^{17}$. However, in this study, we observed an increase in the monoclinic content and the laboratory procedures were somewhat complex. Obtaining powders for this method requires specialized equipment, such as a ball mill and extremely fine mesh sieves. The powder-alcohol mixture must be used immediately as decantation occurs at the bottom of the glass, which can clog the fine canaliculus of the airbrush. In addition, if the jets are not well standardized, they can cause a very thick deposition, which could influence the adaptation of crowns. Such difficulties may not have been found in the original method because the author used an unsintered zirconia powder ${ }^{17}$, whereas in the present study, the powder was soured from a pre-sintered material. Another difference lies in the size of the particles. Aboushelib ${ }^{17}$ (2012) used a granulated powder with particle size in the range of 7 to $12 \mu \mathrm{m}$, while in the present study, particles of up to $45 \mu \mathrm{m}$ were used due to the unavailability of smaller mesh sieves. 
Even thought the FS treatment resulted in higher bond strength values when compared to the untreated group, it had low bond values, being lower than the other surface treatments, as AAP. These findings are not in agreement with Aboushelib ${ }^{17}$ (2012), in which FS resulted in bond strengths similar to those obtained with alumina-particle treatment. However, a direct comparison between these studies is not recommended, because in the AAP group, besides air abrasion with alumina particles, a primer containing multiple bond promoters was applied.

The group in which silica nanofilms were deposited also exhibited higher adhesive strengths than the untreated group, but much lower values than the TBS group. These findings are not in agreement with those of Druck et al. ${ }^{18}$ (2015), who reported high bond strengths (similar to TBS) for $5 \mathrm{~nm}$ silica deposition treatment. Furthermore, the deposition of silica nanofilms did not result in significant differences in the topography compared to the specimens on which nanofilms were not deposited (AS and FS) (Figure 3, images C-D and G-H).

An attempt was made in the FSSN group to combine micromechanical retention with chemical bonding by conducting fusion sputtering followed by the deposition of a silica nanofilm. It resulted in a lower adhesive strength than the TBS group, in spite of the combinatorial action of the two treatments.

The application of a primer containing a phosphoric acid methacrylate ester monomer (FSSNP group) did not result in an increased bond strength to the resin cement when compared to the group in which a silane agent was applied (FSSN). Without previous air abrasion with alumina particles (AAP group), the primer application was not as effective as TBS, even though it was associated with two other treatments (FS and SN), from which we can conclude that its use is of secondary importance.

Microshearing conditions were chosen for testing the bond strength, as these tests are easier to conduct than microtensile tests; it must be emphasized that the ceramic is extremely resistant to cutting ${ }^{19}$. However, one of the limitations of this test approach is that non-homogeneous stresses may be developed at the adhesive zone and interfaces. Further, cohesive fractures can occur in the materials ${ }^{18}$. Despite these features, only minor fracture characteristics were observed in the cement on the side opposite to load application, indicating a predominantly adhesive failure in the groups with high bond strengths. Oyagüe et al. ${ }^{25}$ (2009) considered this type of failure preferable to fully adhesive failure, because the latter is often associated with low values of bond strength, as observed in the present study.

Another limitation of the microshear test is the inclusion of air bubbles and marginal cracks during insertion of cement into the mold, leading to a premature failure ${ }^{19}$. In the present study, microcylinders that exhibited pre-test failures were excluded from statistical analysis. The use of starch tubes, easily removed after $24 \mathrm{~h}$ of immersion in water, allowed the preparation of uniform microcylinders without concentrated stresses prior to testing ${ }^{19}$.

No aging procedure was performed; thus, the bond durability could not be assessed. Consequently, our findings should be considered with caution. New studies should be conducted for parameter improvements to make fusion sputtering and the deposition of silica nanofilms alternative treatments to silicatization followed by silanization. 
The following conclusions were drawn:

1. Tribochemical silica coating and air abrasion with alumina particles + primer containing multiple bond promoters resulted in the highest bond strength between resin cement and $\mathrm{Y}$-TZP.

2. Fusion sputtering (zirconia particles) and silica nanofilm deposition resulted in low bond strength values. However, when these methods are applied in combination and with a primer (FSSN and FSSNP), higher bond strengths might be achievable.

3. Low bond strengths are obtained with control zirconia, which is not subjected to any treatment.

\section{Acknowledgements}

The authors state there are no conflicts of interest. We thank Ivoclar Vivadent for donating the ceramic material.

\section{REFERENCES}

1. Casucci A, Monticelli F, Goracci C, Mazzitelli C, Cantoro A, Papacchini F, et al. Effect of surface pre-treatments on the zirconia ceramic-resin cement microtensile bond strength. Dent Mater. 2011 Oct;27(10):1024-30. doi: 10.1016/j.dental.2011.07.002.

2. Da Silva EM, Miragaya L, Sabrosa CE, Maia LC. Stability of the bond between two resin cements and an yttria-stabilized zirconia ceramic after six months of aging in water. J Prosthet Dent. 2014 Sep;112(3):568-75. doi: 10.1016/j.prosdent.2013.12.003

3. Yun J-Y, Ha S-R, Lee J-B, Kim S-H. Effect of sandblasting and various metal primers on the shear bond strength of resin cement to Y-TZP ceramic. Dent Mater. 2010 Jul;26(7):650-8. doi: 10.1016/j.dental.2010.03.008.

4. Cheung GCK, Botelho MG, Matinlinna JP. Effect of surface treatments of zirconia ceramics on the bond strength to resin cement. J Adhes Dent. 2014 Feb;16(1):49-56. doi: 10.3290/j.jad.a30753.

5. Passos SP, May LG, Barca DC, Özcan M, Bottino MA, Valandro LF. Adhesive quality of self-adhesive and conventional adhesive resin cement to Y-TZP ceramic before and after long-term storage and thermal cycling. Oper Dent. 2010 Nov;35:689-96. doi: 10.2341/10-157-L.

6. Inokoshi M, VanMeerbeek B. Adhesively luted zirconia restorations: why and how? J Adhes Dent. 2014 Jun;16(3):294. doi: 10.3290/j.jad.a32188.

7. Örtorp A, Kihl ML, Carlsson GE. A 5-year retrospective study of survival of zirconia single crowns fitted in a private clinical setting. J Dent. 2012 Jun;40(6):527-30. doi: 10.1016/j.jdent.2012.02.011.

8. Al-Amleh B, Lyons K, Swain M. Clinical trials in zirconia: A systematic review. J Oral Rehabil. 2010 Aug;37(8):641-52. doi: 10.1111/j.1365-2842.2010.02094.x.

9. Perdigão J, Fernandes SD, Pinto AM, Oliveira FA. Effect of artificial aging and surface treatment on bond strengths to dental zirconia. Oper Dent. 2013 Mar-Apr;38(2):168-76. doi: 10.2341/11-489-L.

10. Zhang Y, Lawn BR, Malament KA, Van Thompson P, Recon ED. Damage accumulation and fatigue life of particle-abraded ceramics. Int J Prosthodont. 2006 Sep-0ct;19(5):442-8. 
11. Amaral M, Cesar PF, Bottino MA, Lohbauer U, Valandro LF. Fatigue behavior of $Y$-TZP ceramic after surface treatments. J Mech Behav Biomed Mater. 2016 Apr;57:149-56. doi: 10.1016/j.jmbbm.2015.11.042.

12. Anami LC, Lima JMC, Valandro LF, Kleverlaan CJ, Feilzer AJ, Bottino MA. Fatigue resistance of $y$-tzp/porcelain crowns is not influenced by the conditioning of the intaglio surface. Oper Dent. 2016 Jan-Feb;41(1):E1-12. doi: 10.2341/14-166-L.

13. Campos F, Valandro LF, Feitosa SA, Kleverlaan CJ, Feilzer AJ, de Jager N, et al. Adhesive cementation promotes higher fatigue resistance to zirconia crowns. Oper Dent. 2017 Mar/Apr;42(2):215-224. doi: 10.2341/16-002-L.

14. Scherrer SS, Cattani-Lorente M, Vittecoq E, de Mestral F, Griggs JA, Wiskott HW. Fatigue behavior in water of Y-TZP zirconia ceramics after abrasion with $30 \mu \mathrm{m}$ silica-coated alumina particles. Dent Mater. 2011 Feb;27(2):e28-42. doi: 10.1016/j.dental.2010.10.003.

15. Pereira GKR, Venturini AB, Silvestri T, Dapieve KS, Montagner AF, Soares FZM, et al. Low-temperature Degradation of Y-TZP Ceramics: A Systematic Review and Meta-analysis. J Mech Behav Biomed Mater. 2015 Mar;55:151-163. doi: 10.1016/j.jmbbm.2015.10.017.

16. Cura C, Özcan M, Isik G, Saracoglu A. Comparison of alternative adhesive cementation concepts for zirconia ceramic: glaze layer vs zirconia primer. J Adhes Dent. 2012 Feb;14(1):75-82. doi: 10.3290/j.jad.a21493.

17. Aboushelib, MN. Fusion sputtering for bonding to zirconia-based materials. J Adhes Dent. 2012 Aug;14(4):323-8. doi: 10.3290/j.jad.a25684.

18. Druck CC, Pozzobon JL, Callegari GL, Dorneles LS, Valandro LF. Adhesion to Y-TZP ceramic: study of silica nanofilm coating on the surface of Y-TZP. J Biomed Mater Res B Appl Biomater. 2015 Jan;103(1):143-50. doi: 10.1002/jbm.b.33184.

19. Tedesco TK, Montagner AF, Skupien JA, Soares FZ, Susin AH, Rocha RO. Starch tubing: an alternative method to build up microshear bond test specimens. J Adhes Dent. 2013 Aug;15(4):311-5. doi: 10.3290/j.jad.a28602.

20. Garvie RC, Nicholson PS. Phase analysis in zirconia systems. J Am Ceram Soc. 1972 Jun;55(6):303-5.

21. Toraya H, Yoshimura M, Somiya S. Calibration Curve for Quantitative Analysis of the Monoclinic-Tetragonal ZrO 2 System by X-ray Diffraction. J Am Ceram Soc. 1984 Jun;67(6):C119-21.

22. Inokoshi M, De Munck J, Minakuchi S, Van Meerbeek B. Meta-analysis of Bonding Effectiveness to Zirconia Ceramics. J Dent Res. 2014 Feb;93(4):329-34.

23. Subaşi MG, Demir N, Kara Ö, Ozturk AN, Özel F. Mechanical properties of zirconia after different surface treatments and repeated firings. J Adv Prosthodont. 2014 Dec;6(6):462-7.

24. Karakoca S, Yilmaz H. Influence of surface treatments on surface roughness, phase transformation, and biaxial flexural strength of Y-TZP ceramics. J Biomed Mater Res B Appl Biomater. 2009 Nov;91(2):930-7. doi: 10.1002/jbm.b.31477.

25. Oyagüe RC, Monticelli F, Toledano M, Osorio E, Ferrari M, Osorio R. Effect of water aging on microtensile bond strength of dual-cured resin cements to pre-treated sintered zirconium-oxide ceramics. Dent Mater. 2009 Mar;25(3):392-9. doi: 10.1016/j.dental.2008.09.002. 